\title{
Effect of Femtosecond-laser Irradiation on Magnetic and Optical Properties in Au- and Fe-doped Transparent Glass
}

\author{
Seisuke NAKASHIMA ${ }^{* 1,{ }^{* 2}}$, Koji SUGIOKA ${ }^{* 1}$, Katsumi MIDORIKAWA ${ }^{* 1}$, and Kohki MUKAI ${ }^{{ }^{22}}$ \\ ${ }^{* 1}$ Advanced Science Institute, RIKEN, 2-1 Hirosawa, Wako, Saitama 351-0198, Japan \\ E-mail:nakashima@ynu.ac.jp \\ ${ }^{* 2}$ Yokohama National University, 79-5 Tokiwadai, Hodogaya-ku, Yokohama-shi, Kanagawa 240- \\ 8501, Japan
}

\begin{abstract}
We have investigated an effect of near-infrared femtosecond- (fs-) laser irradiation on magnetic and optical properties in Au- and Fe-doped transparent glasses. A fs-laser beam with a wavelength of $775 \mathrm{~nm}$ was focused $1 \mathrm{~mm}$ below the surfaces of glass samples. This produces absorption peaks due to the formation of hole-trap centers in the irradiated region, which changes into different peaks originated from localized surface plasmon absorption after annealing at $450^{\circ} \mathrm{C}$. It is presumed that precipitation of $\mathrm{Au}$ nanoparticles occurs as results of photoreduction of $\mathrm{Au}$ ions and agglomeration by the thermal atomic diffusion. In addition, enhancement of magnetization in the localized area inside the glass was realized after laser irradiation and subsequent annealing. Ferrimagnetic behaviors were increased in the $M-H$ curve even at room temperature, whereas the diamagnetic component dominated in the $M-H$ curve of the as-prepared glass sample. The irradiated and annealed glass sample also exhibited superparamagnetic blocking in the temperature dependence of the magnetization with a blocking temperature higher than room temperature. These changes in magnetic properties are explainable in terms of the local crystallization of ferrimagnetic nanoparticles, such as magnetite, induced by fs-laser irradiation and annealing. Transmission-electron-microscopy observations support our considerations that $\mathrm{Au}$ nanoparticles and ferrimagnetic spinel-phase nanoparticles were simultaneously and separately crystallized in the focused region of the fs-laser beam. DOI:10.2961/jlmn.2012.02.0015
\end{abstract}

Keywords: Femtosecond laser, Ferrimagnetic nanoparticle, Superparamagnet, Plasmonic material, Localized surface plasmon resonance

\section{Introduction}

Ultrashort-pulse-laser induced synthesis of nanocrystalline materials in transparent glasses has attracted considerable attention for its potential applications in a variety of devices, such as nonlinear optical devices, plasmonic devices, and magnetic recording devices. This is because various physical properties, including optical, electrical, mechanical, and magnetic properties of nanocrystalline materials can be drastically controlled by changing the synthesis conditions [1-7]. In this viewpoint, one of the most significant features of femtosecond (fs) lasers is direct three-dimensional (3D) processing method $[8,9]$. However, studies on space-selective modification of magnetic nanocrystalline materials by fs laser irradiation have been rarely reported. Generally, ultra-short pulse lasers have been utilized for sensing the fast dynamics of magnetic transitions. In such systems, magnetic transitions or changes are transient and relaxed rapidly when the excitation light is turned off $[10,11]$. However, some groups have exceptionally observed persistent magnetic phase transitions caused by fs-laser irradiation $[12,13]$. In these cases, structural change is essential for achieving a persistent change in the magnetism. In our previous study, we achieved enhancement of magnetization in localized area inside a Fe-oxidedoped transparent paramagnetic glasses by a fs-laser irradiation. This magnetic transition presumably originates from a precipitation of ferrimagnetic $\mathrm{Fe}_{3} \mathrm{O}_{4}$ nanoparticles, that occurs as a result of photoreduction of $\mathrm{Fe}^{3+}$ ions and crystallization by subsequent anneal process [14]. The laser processing technology has the potential to be used for patterning ferromagnetic materials. In order to develop this technique for application to $3 \mathrm{D}$ high-density magnetic recording devices or magneto-optical microdevices, we have tried to crystalize different functional crystals such as magnetic and plasmonic nanoparticles. In this paper, we have fabricated a glasses doped with $\mathrm{Au}$ ions and $\mathrm{Fe}$ ions and investigated the effect of fs-laser irradiation on magnetic and optical properties.

\section{Experimental procedures}

Soda lime silicate glass co-doped with Au and Fe-oxide was used in this study. Its composition was $0.01 \mathrm{HAuCl}_{4} \cdot 0.05 \mathrm{Fe}_{2} \mathrm{O}_{3} \cdot 70 \mathrm{SiO}_{2} \cdot 20 \mathrm{Na}_{2} \mathrm{O} \cdot 10 \mathrm{CaO} \quad(\mathrm{mol} / \%)$. We also doped the glass with $0.1 \mathrm{~mol} \% \mathrm{Al}$ to reduce the $\mathrm{Fe}^{3+}$ ion effectively. Reagent-grade $\mathrm{SiO}_{2}, \mathrm{Na}_{2} \mathrm{CO}_{3}, \mathrm{CaCO}_{3}$, $\mathrm{HAuCl}_{4}, \mathrm{Fe}_{2} \mathrm{O}_{3}$ (hematite), and $\mathrm{Al}$ were used as starting materials. A mixture of these powders was melted in a platinum crucible at $1550{ }^{\circ} \mathrm{C}$ for $1 \mathrm{~h}$ in air. The melt was then quenched into a transparent glass by casting it onto a stainless-steel plate at room temperature. The polished glass samples with the thickness of about $3 \mathrm{~mm}$ were used.

We utilized a near-infrared fs laser (Clark-MXR, CPA2001; wavelength: $775 \mathrm{~nm}$; repetition rate: $1 \mathrm{kHz}$; pulse widths: $150 \mathrm{fs}$ (full width at half maximum)). The laser 
beam was collimated by a 3-mm-diameter aperture and was focused $1 \mathrm{~mm}$ below the glass surface using a $\times 20$ microscope objective with a numerical aperture of 0.46 . The laser beam had an average power of $20 \mathrm{~mW}$ and the beam was focused to produce a $2.7-\mu \mathrm{m}$-diameter spot. The samples were placed horizontally on a PC-controlled $X Y$ translation stage and translated in the $X$ direction at a scan speed of $1000 \mu \mathrm{m} / \mathrm{s}$. Laser-irradiated lines were repeatedly scanned with a pitch of $5 \mu \mathrm{m}$ in the $Y$ direction, producing a square irradiated area with 4-mm-long sides. After the fslaser irradiation, the glass samples were annealed at $450{ }^{\circ} \mathrm{C}$ for 30 minutes and additionally 30 and 60 minutes (totally 60 and 90 minutes). For comparison, we also annealed a non-irradiated glass sample at $450{ }^{\circ} \mathrm{C}$ and $550{ }^{\circ} \mathrm{C}$. The absorption spectra of glass samples were measured using a spectrophotometer (JASCO V-570). All magnetic measurements were performed using a superconducting quantum interference device (SQUID) magnetometer (Quantum Design, MPMS-XL).

\section{Results and discussions}

\subsection{Optical properties}

As a preliminary experiment, the glass samples were annealed at 450 and $550{ }^{\circ} \mathrm{C}$ without fs-laser irradiation. Figure 1 shows the absorption spectra of the as-prepared sample and those annealed at each temperature for $30 \mathrm{~min}$. The photographs for each sample were exhibited in the inset. The as-prepared glass sample is highly transparent at visible wavelengths, i.e., wavelengths longer than $340 \mathrm{~nm}$ (black curve). This high transparency of glass doped with transition metal $\mathrm{Fe}$ ions is ascribed to the small absorption cross section of trivalent Fe ions, which is due to all $d$ - $d$ transitions being spin forbidden in a high-spin configuration. For the sample after anneal at $450{ }^{\circ} \mathrm{C}$ (red dotted curve), the absorption spectrum is almost equivalent to that for the as-prepared glass sample. In appearance, the optical transparency was maintained in this sample. In the case of the higher temperature anneal (red solid curve), however, we found a distinct absorption peak at around $514 \mathrm{~nm}$, which is due to a red coloration over the whole sample. This change originates from the precipitation of Au nanoparticles, which was generated as a result of reduction of $\mathrm{Au}^{3+}$ ions and oxidation of $\mathrm{Al}$ atoms. $\mathrm{Au}^{3+}$ ions received three electrons from $\mathrm{Al}$ atoms, at the same time the reduced $\mathrm{Au}$ atoms easily aggregated and became nano-sized particles during the high temperature annealing $[15,16]$. In other words, single anneal process at the lower temperature $\left(450{ }^{\circ} \mathrm{C}\right)$ does not cause reduction of $\mathrm{Au}^{3+}$ ions, even in the presence of $\mathrm{Al}$ atoms.

Absorption spectra were measured for the laserirradiated and annealed glass samples. Figure 2 displays the difference in spectra between the as-prepared and the each sample. The inset shows photographs of the laserirradiated sample and that annealed at $450{ }^{\circ} \mathrm{C}$ for $90 \mathrm{~min}$. As can be seen from the top and side-view of the laserirradiated sample, the laser beam was focused on the localized region, which turned brown. This color change is attributed to the broad absorption peaks at about 430 and 620 $\mathrm{nm}$ in spectrum (a). These absorptions are due to hole-trap centers (e.g., $\mathrm{HC}_{1}$ and $\mathrm{HC}_{2}$ ) at nonbridging oxygens. $\mathrm{HC}_{1}$ and $\mathrm{HC}_{2}$ are holes trapped at the nonbridging oxygen in the

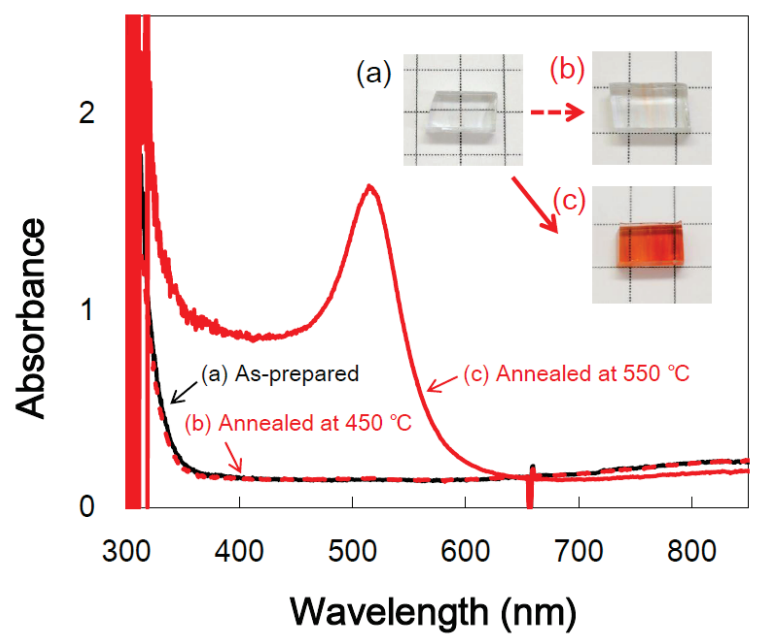

Fig. 1 Optical absorption spectra of as-prepared glass sample (black curve) and that annealed at $450{ }^{\circ} \mathrm{C}$ (red dotted curve) and at $550{ }^{\circ} \mathrm{C}$ (red solid curve) without fs-laser irradiation. The inset shows photographs of the samples.

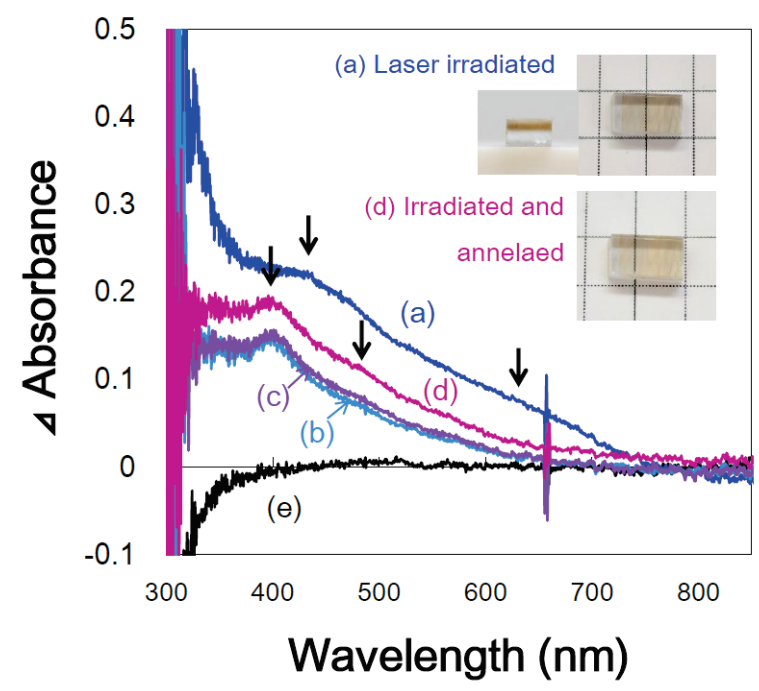

Fig. 2 Difference spectra in absorption spectra between asprepared and fs-laser irradiated glass samples; the irradiated (a), the irradiated and annealed at $450{ }^{\circ} \mathrm{C}$ for $30 \mathrm{~min}(\mathrm{~b})$, that for $60 \mathrm{~min}(\mathrm{c})$, that for $90 \mathrm{~min}$, and only annealed at $450{ }^{\circ} \mathrm{C}$ for $30 \mathrm{~min}$ (e). The inset shows photographs of the samples.

$\mathrm{SiO}_{4}$ polyhedron with two and three nonbridging oxygens, respectively $[6,17,18]$. Free electrons were driven out from the $2 p$ orbital of the nonbridging oxygen due to the multiphoton absorption of the incident photons. In the present case, the doped Al atoms supply electrons to the holes centers on the nonbridging oxygens and form covalent bonds with the oxygens, leading that the free electrons from the oxygens become slightly stable. Hence, $\mathrm{Au}^{3+}$ ions would be easily reduced into atomic state by trapping these free electrons. By the following anneal process, the $\mathrm{Au}$ atoms agglomerate and $\mathrm{Au}$ nanoparticles will be crystalized In the spectrum for the irradiated and annealed sample (b), the peaks due to hole-trap centers disappeared and another broad absorption peak can be observed at about $485 \mathrm{~nm}$, which is presumably due to localized surface plasmon absorption of the Au nanoparticles. The peak shift from 514 $\mathrm{nm}$ suggests that the precipitated $\mathrm{Au}$ nanoparticles are 
much smaller than those obtained by the annealing at 550 ${ }^{\circ} \mathrm{C}$. As increasing the annealing time, the absorption peaks become more intense, indicating the increase in the number of Au nanoparticles (see spectra (c) and (d)). In the meanwhile, a small absorption band was also observed at around $400 \mathrm{~nm}$, the origin of which is unclear. The optical modification has not been definitely induced by the single anneal process at the same temperature, $\sim 450^{\circ} \mathrm{C}$ (spectrum (e)).

In our previous paper, we investigated an effect of fslaser irradiation of $\mathrm{Fe}^{3+}$-doped glass on the optical and magnetic properties [14]. Some $\mathrm{Fe}^{3+}$ ions, which disperse in the irradiated region, successfully trap the free electrons and can be reduced to the lower valence state, such as $\mathrm{Fe}^{2+}$ [19]. Therefore, a similar phenomenon, namely, reduction of $\mathrm{Fe}^{3+}$ ions, is expected to occur also in the present case. As mentioned in our previous paper, reduction of $\mathrm{Fe}^{3+}$ induced no change in absorption spectra.

\subsection{Magnetic properties}

To estimate the change of magnetic properties, we measured the external magnetic field dependences of the magnetization at room temperature. The inset of Fig. 3 shows raw magnetization data for as-prepared sample (black circles), irradiated sample (blue closed circles), and irradiated and annealed samples (blue open symbols). The as-prepared sample had a very low saturation magnetization, $M_{\mathrm{s}}$, indicating that the sample behaves as a paramagnet at room temperature, because magnetic interactions between $\mathrm{Fe}$ ions that are highly dispersed in the glass are significantly weak. Hence, the negligible contribution to the $M_{s}$ is presumably due to the local magnetic interaction between slightly aggregated $\mathrm{Fe}$ ions. On the other hand, the irradiated and annealed samples have relatively higher magnetization compared to the as-prepared sample. Since the ferromagnetic contributions are saturated at high magnetic fields, the negative gradient of the linear diamagnetic component due to the non-magnetic glass matrix can be subtracted as a background. And then, the difference in the magnetization after background subtraction between asprepared and the other samples are plotted in the main flame of Fig. 3. For comparison, those between the asprepared and the only annealed samples were also shown (red symbols). The increase in $M_{s}$ for the irradiated and annealed samples is much larger than that for the annealed sample, which suggests that the fs-laser irradiation is necessary to enhance the magnetization. As is well known, a focused fs laser beam does not affect areas far from the focal point. It is thus reasonable to assume that the change in the magnetization is restricted to the irradiated region. Our previous paper has reported that the precipitation of ferrimagnetic $\mathrm{Fe}_{3} \mathrm{O}_{4}$ nanoparticles induced by fs-laser irradiation and post anneal process is a possible origin of the enhancement of magnetization. Assuming that the magnetic property of the glass is independent of the other dopants, $\mathrm{Al}$ and $\mathrm{Au}$ ion, a similar situation will be applied to the present case. Figure 4(a) shows temperature dependences of zero-field-cooled (ZFC) and field-cooled (FC) magnetizations, $M_{\mathrm{ZFC}}$ and $M_{F C}$ measured at a dc magnetic field of 500 Oe for the as-prepared and the laser-irradiated samples. While the as-prepared glass is almost completely paramagnetic even at low temperature, the $M_{\mathrm{ZFC}}$ deviates from the $M_{F C}$ below room temperature in the case of the irradiated

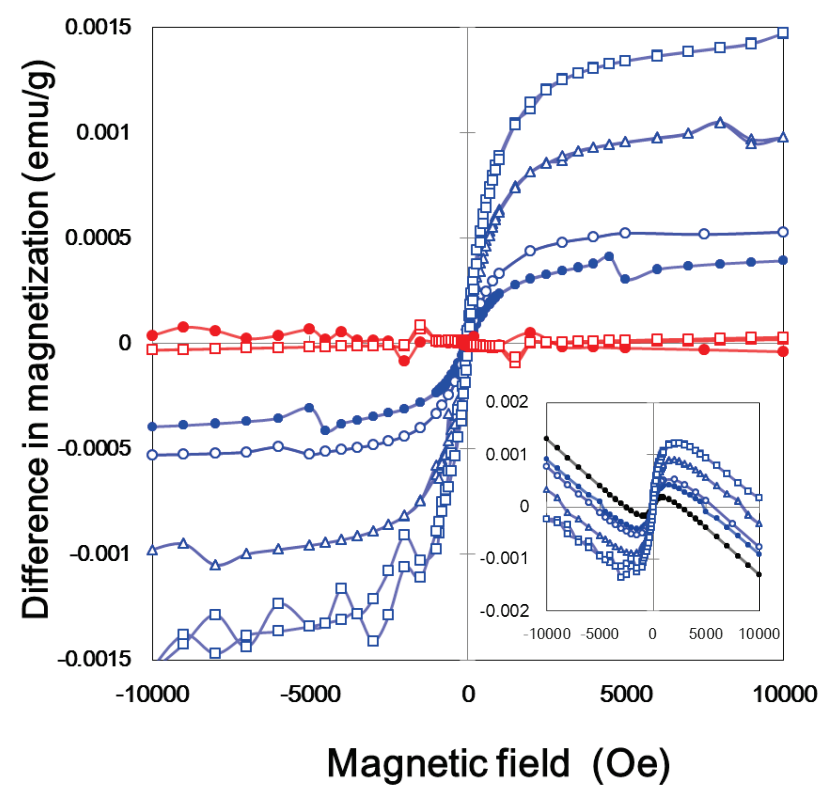

Fig. 3 Inset shows external magnetic field dependences of magnetization at room temperature for the as-prepared (black $\bullet$ ), the irradiated (blue $\bullet$ ), the irradiated and annealed at $450{ }^{\circ} \mathrm{C}$ for $30 \mathrm{~min}$ (blue $\circ$ ), that for $60 \mathrm{~min}$ (blue $\Delta$ ), that for $90 \mathrm{~min}$ (blue $\square$ ). Main plot shows difference in magnetization between the as-prepared and each sample. For comparison, the only annealed samples at $450{ }^{\circ} \mathrm{C}\left(\right.$ red $\bullet$ ) and $550{ }^{\circ} \mathrm{C}$ (red $\square$ ) are also shown.
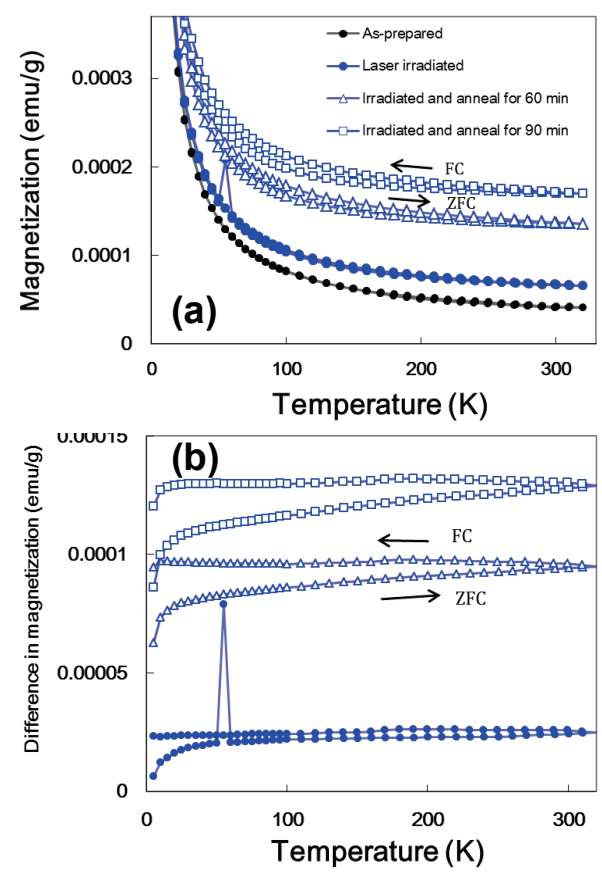

Fig. 4 (a) Temperature dependences of magnetization under an external field of 500 Oe for the as-prepared (black O), the irradiated (blue $\mathbf{O}$ ), the irradiated and annealed for $60 \mathrm{~min}$ (blue $\Delta$ ), and that for $90 \mathrm{~min}$ (blue $\square$ ). (b) Difference in magnetization between the asprepared and each sample.

samples. The differences in magnetization between asprepared and the laser-irradiated samples as a function of temperature are also presented in Fig. 4(b). The irradiated and annealed sample exhibits superparamagnetic blocking above room temperature, which is typically observed in 
ferromagnetic nanoparticles with interparticle dipole interactions $[11,20]$. It should be noted that the broad and prolonged blocking phenomenon stems from an inhomogeneous dispersion of nanoparticles. In addition, the enhanced magnetization curve for the irradiated and annealed sample has a small coercive field of $\sim 40 \mathrm{Oe}$, which also implies that high magnetization originates from ferrimagnetic $\mathrm{Fe}_{3} \mathrm{O}_{4}$ nanoparticles.

The $M_{s}$ measured for the laser irradiated and annealed glass (90 min) is 3.7 times as high as that for the asprepared glass. Taking it into account that about $24 \%$ of bulk sample is colored and changes in magnetization, however, an enhancement factor in $M_{s}$ can be estimated to be 12 at least. In the case the annealing for $60 \mathrm{~min}$, the factor is calculated to be 9.1. This value is almost equivalent to the case of the Fe-singly-doped glass in our previous paper. It should be mentioned that the actual magnetization enhancement could be much greater if the region in which ferrimagnetic nanoparticles crystalized was underestimated. At present, it is impossible to perform an accurate estimation because the change of magnetism is presumably spatially inhomogeneous. The intensity of the focused fs-laser beam decreases with distance from the focal point. Therefore, the spatial density of $\mathrm{Fe}_{3} \mathrm{O}_{4}$ nanocrystals will vary from area to area depending on the beam intensity distribution.

\subsection{Nanostructures}

In order to investigate the nanostructure induced by fslaser in the glass, direct observations were performed using TEM. A plan-view TEM sample was fabricated by an Ar milling process. Figure 5 shows bright field TEM images for the irradiated and annealed glass sample. We have found two kinds of nanoparticles. One (Fig. 5(a)) is 5 to 10 $\mathrm{nm}$ dark contrasted nanoparticles, which is presumably metallic $\mathrm{Au}$ nanoparticles with localized surface plasmon resonance, although a composition analysis has not been carried out yet. The other (Fig.5(b)) is 5 to $18 \mathrm{~nm}$ crystalline phase, which indicates a spinel-oxide typed diffraction pattern shown in Fig. 5(c). As discussed before, trivalent $\mathrm{Fe}$ ions distributed at around the focal position of the focused laser beam are locally and partly reduced to divalent ions. During the laser irradiation with a fs laser, atomic diffusion of $\mathrm{Fe}$ ions occurs due to the heat gradient generated near the focal point [21], resulting in locally Fe-highdensity regions. In this case, Fe-oxide phase would be easily crystallized by external thermal stimulation, such as low temperature annealing. Therefore, it is speculated that the possibly crystalized spinel phase is ferrimagnetic $\mathrm{Fe}_{3} \mathrm{O}_{4}$ which is the most stable crystalline phase in the mixed valence states of Fe ions. Measurements of complementary experiments such as nano-beam diffraction or composition analysis were remaining at present.

\section{Conclusions}

We demonstrated spatially selective control of optical property and magnetism by focusing an infrared fs laser beam inside transparent $\mathrm{Au}$ - and $\mathrm{Fe}$-doped glasses at room temperature and subsequent annealing at $450{ }^{\circ} \mathrm{C}$. The irradiated area turns brown after laser irradiation and then

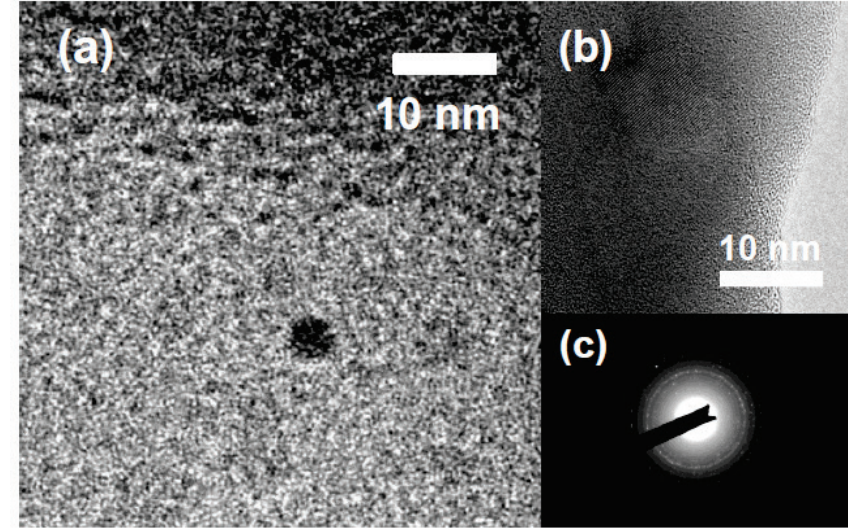

Fig. 5 (a) (b) bright field TEM image for the irradiated and annealed glass sample. (c) Electron-beam-diffraction pattern for the sample.

slightly fades after annealing. The absorption after irradiation is attributed to the formation of hole-trap centers and the generated free electrons locally reduces not only $\mathrm{Fe}^{3+}$ ions to $\mathrm{Fe}^{2+}$ ions but also $\mathrm{Au}$ ions to $\mathrm{Au}$ atoms, resulting in localized surface plasmon absorption of $\mathrm{Au}$ nanoparticles. The irradiated and annealed glass sample had a higher saturation magnetization than the as-prepared glass, which is due to a ferrimagnetic phase consisting of spinel $\mathrm{Fe}_{3} \mathrm{O}_{4}$ precipitated and grown by annealing. Nucleation and growth of nanocrystalline phase is confined in local regions with high $\mathrm{Fe}$ concentrations formed by an atomic diffusion of Fe ions as a result of high energy deposition by focused fs laser. In this case, magnetic ordering is limited inside the ferrimagnetic nanoparticles, although the magnetic interactions operate even at room temperature. The enhancement factor of the saturation magnetization is estimated to be as high as 12 . These results can be explained in terms of co-precipitation of $\mathrm{Au}$ nanoparticles and ferrimagnetic spinel-oxide phase nanoparticles in the focused region of the fs-laser beam, which is supported by TEM observations. In the near future, these kinds of materials are practically applicable to extremely novel devices that combine magneto-optical effect with plasmonics [22, 23].

\section{Acknowledgments}

This work was financially supported by a Grant-in-Aid for Science Research (No. 20860095) from the Ministry of Education, Culture, Sports, Science, and Technology (MEXT), Japan, by the Murata Foundation, and by the Nippon Sheet Glass Foundation.

\section{References}

[1] J. Qui, , M. Shirai, T. Nakaya, J. Si, X. Jiang, C. Zhu, and K. Hirao, Appl. Phys. Lett. 81, (2002) 3040. (Journals)

[2] K. Miura, J. Qiu, T. Mitsuyu, K. Hirao, Opt. Lett. 25, (2000) 408. (Journals)

[3] B. Zhu, et al., Opt. Express 15, (2007) 6069. (Journals)

[4] S. Kanehira, K. Miura, K. Fujita, K. Hirao, J. Si, N. Shibata and Y. Ikuhara, Appl. Phys. Lett. 90, (2007) 163110. (Journals)

[5] Q. Zhao, J. Qiu, X. Jiang, C. Zhao, and C. Zhu, J. Appl. Phys. 96, (2004) 7122. (Journals) 
[6] K. M. Davis, K. Miura, N. Sugimoto, and K. Hirao, Opt. Lett. 21, (1996) 1729. (Journals)

[7] S. Nakashima, K. Fujita, A. Nakao, K. Tanaka, Y. Shimotsuma, K. Miura and K. Hirao, Appl. Phys. A 94, (2009) 83. (Journals)

[8] K. Miura, J. Qiu, H. Inouye, T. Mitsuyu, K. Hirao, Appl. Phys. Lett. 71, (1997) 3329. (Journals)

[9] Y. Cheng, K. Sugioka, K. Midorikawa, M. Masuda, K. Toyoda, M. Kawachi, and K. Shihoyama, Opt. Lett. 28, (2003) 1144. (Journals)

[10] M. Rini, A. Cavalleri, R. W. Schoenlein, R. López, L. C. Feldman, R. F. Haglund, Jr., L. A. Boatner and T. E. Haynes, Opt. Lett. 30, (2005) 558. (Journals)

[11] S. Iwai, S. Tanaka, K. Fujinuma, H. Kishida, H. Okamoto, and Y. Tokura, Phys. Rev. Lett. 88, (2002) 057402. (Journals)

[12] M. Matsubara, Y. Okimoto, T. Ogasawara, S. Iwai, Y. Tomioka, H. Okamoto, and Y. Tokura, Phys. Rev. B 77, (2008) 094410. (Journals)

[13] J. H. Kim, J. Kim, S. U. Lim, C. K. Kim, C. S. Yoon1, G. J. Lee, and Y. P. Lee, J. Appl. Phys. 99, (2006) 08G311. (Journals)
[14] S. Nakashima, K. Sugioka, and K. Midorikawa, Appl. Phys. A, in press (2011). (Journals)

[15]X. Hu, Q. Zhao, X. Jiang, C. Zhu, J. Qiu, Sol. State Comm. 138, (2006) 43. (Journals)

[16] S. Qu, Y. Zhang, H. Li, J. Qiu, C. Zhu, Opt. Materials 29, (2006) 259. (Journals)

[17]A. Bishay, J. Non-Cryst. Solids 3, (1970) 54 (Journals)

[18] J. Qiu, X. Jiang, Q. Zhao, C. Zhu, H. Inoue, J. Si, and K. Hirao, Opt. Lett. 29, (2004) 370. (Journals)

[19] J. Qiu, C. Zhu, T. Nakaya, J. Si, F. Ogura, K. Kojima, and K. Hirao, Appl. Phys. Lett. 79, (2001) 3567. (Journals)

[20] S. Nakashima, K. Fujita, K. Tanaka, and K. Hirao, J. Phys.: Condensed Matter 17, (2005) 137. (Journals)

[21] K. Miura, K. Hirao, Y. Shimotsuma, M. Sakakura, and S. Kanehira, Appl. Phys. A 93, (2008) 183. (Journals)

[22] P. K. Jain, Y. Xiao, R. Walsworth, and A. E. Cohen, Nano lett. 9, (2009) 1644. (Journals)

[23] L. Wang, K. Yang, C. Clavero, A. J. Nelson, K. J. Carroll, E. E. Carpenteer, and R. A. Lukaszew, J. Appl. Phys. 107, (2010) 09B303. (Journals)

(Received: July 26, 2011, Accepted: April 24, 2012) 